\title{
Paired analysis of tumor mutation burden calculated by targeted deep sequencing panel and whole exome sequencing in non-small cell lung cancer
}

\author{
Sehhoon Park, ${ }^{1, \#}$, Chung Lee, ${ }^{2, \#}$, Bo Mi Ku ${ }^{1}$, Minjae Kim², Woong-Yang Park ${ }^{2,3}$, Nayoung K. D. Kim ${ }^{2, *} \mathcal{E}^{*}$ Myung-Ju Ahn ${ }^{1, *}$ \\ ${ }^{1}$ Division of Hematology-Oncology, Department of Medicine, Samsung Medical Center, Sungkyunkwan University School of Medicine, \\ Seoul 06351, ${ }^{2}$ Geninus Inc., Seoul 05836, ${ }^{3}$ Samsung Genome Institute, Samsung Medical Center, Seoul 06351, Korea
}

\begin{abstract}
Owing to rapid advancements in NGS (next generation sequencing), genomic alteration is now considered an essential predictive biomarkers that impact the treatment decision in many cases of cancer. Among the various predictive biomarkers, tumor mutation burden (TMB) was identified by NGS and was considered to be useful in predicting a clinical response in cancer cases treated by immunotherapy. In this study, we directly compared the lab-developed-test (LDT) results by target sequencing panel, K-MASTER panel v3.0 and whole-exome sequencing (WES) to evaluate the concordance of TMB. As an initial step, the reference materials $(n=3)$ with known TMB status were used as an exploratory test. To validate and evaluate TMB, we used one hundred samples that were acquired from surgically resected tissues of non-small cell lung cancer (NSCLC) patients. The TMB of each sample was tested by using both LDT and WES methods, which extracted the DNA from samples at the same time. In addition, we evaluated the impact of capture region, which might lead to different values of TMB; the evaluation of capture region was based on the size of NGS and target sequencing panels. In this pilot study, TMB was evaluated by LDT and WES by using duplicated reference samples; the results of TMB showed high concordance rate $\left(R^{2}=0.887\right)$. This was also reflected in clinical samples $(n=100)$, which showed $R^{2}$ of 0.71 . The difference between the coding sequence ratio $(3.49 \%)$ and the ratio of mutations $(4.8 \%)$ indicated that the LDT panel identified a relatively higher number of mutations. It was feasible to calculate TMB with LDT panel, which
\end{abstract}

*Corresponding authors. Nayoung K.D. Kim, Tel: +82-2-6949-6580; Fax: +82-2-6949-6580; E-mail: Nayoung.kim@kr-geninus.com; MyungJu Ahn, Tel: +82-2-3410-3438; Fax: +82-2-3410-1754; E-mail: silk. ahn@samsung.com

${ }^{\#}$ These authors contributed equally to this work.

https://doi.org/10.5483/BMBRep.2021.54.7.045

Received 31 March 2021, Revised 19 April 2021, Accepted 2 June 2021

Keywords: Immunotherapy, Next generation sequencing, Targeted exome sequencing, Tumor mutation burden can be useful in clinical practice. Furthermore, a customized approach must be developed for calculating TMB, which differs according to cancer types and specific clinical settings. [BMB Reports 2021; 54(7): 386-391]

\section{INTRODUCTION}

Next-generation sequencing (NGS) technique has undergone several advancements in recent times. In clinical practice, comprehensive genome profiling is now done extensively with NGS technique because it has a short turnaround time and an acceptable cost of operation $(1,2)$. In particular, cancer patients are being extensively screened with NGS technique. The results are used to devise actionable genomic alteration, which is now an essential step in deciding the preferred mode of treatment (3). As the number of target genes increases, an optimized panel of contents and size have been constructed by targeting genes related to specific cancer types. This has been widely the conventional mode of NGS technique for the past several years $(4,5)$.

The initial treatment decision is usually made after performing NGS technique, which accurately detects following mutations: single nucleotide variation (SNV), insertion and deletions (INDEL), copy number variation (CNV), and fusion. Recently, an immune checkpoint inhibitor (ICl) that targets PD-1 and PD-L1 axis has become the standard mode of treatment for patients with different types of cancer $(6,7)$ including non-small cell lung cancer (NSCLC). Therefore, several efforts are being made to develop a predictive biomarker that measures genomic alteration, which is a daunting task in genomics. Presently, tumor mutation burden (TMB) is considered as a candidate biomarker because several studies have reported that tumors with a high mutation burden are more likely to respond to $\mathrm{ICl}$ treatment (8-10). Interestingly, this finding has also been observed in patients with same types of cancer. In many retrospective studies of NSCLC patients, it has been reported that clinical outcomes of $\mathrm{ICl}$ treatment were better in patients with high mutation burden, which was determined by whole exome sequencing (11). Moreover, this finding was confirmed by evaluating TMB

ISSN: 1976-670X (electronic edition)

Copyright (C) 2021 by the The Korean Society for Biochemistry and Molecular Biology

(c) This is an open-access article distributed under the terms of the Creative Commons Attribution Non-Commercial License (http://creativecommons.org/licenses/by-nc/4.0) which permits unrestricted non-commercial use, distribution, and reproduction in any medium, provided the original work is properly cited. 
with target sequencing panel (12). Therefore, target sequencing panel can be effectively used in cancer treatment: it is not only a companion diagnostic test that detects oncogenic drivers for targeted therapy, but it is also used to determine microsatellite instability (MSI) and TMB for cancer immunotherapy $(13,14)$.

The TMB value indicates the total number of mutations in the analyzed genomic region, and it is reported that there are variations for each type of tumor (15). While assessing the value of $\mathrm{TMB}$, oncologists usually count somatic mutations in the entire exonic region: all the mutations are counted by the whole exome sequencing (WES) method, regardless of whether they are synonymous or non-synonymous in nature $(11,16)$. In contrast, oncologists encounter several challenges while calculating TMB by target sequencing panel. Firstly, there are limited number of genes in the target sequencing panel, so representative value becomes an issue for a small sized panel. Therefore, scientists have suggested using a panel whose size is larger than at least 1 megabase pair $(\mathrm{Mbp})(17,18)$. Secondly, while calculating TMB, scientists use specific methods for evaluating mutation scoring and for defining cutoff levels. These parameters indicate deleterious and clinically significant variants, which are not standardized till date $(19,20)$. These uncertain parameters have generated a controversy in defining high versus low TMBs. Moreover, certain studies show that the difference between carcinomas is obvious, and no cutoff value can be used universally (21). Last but not the least, very few studies have illustrated the prospective clinical benefits of using TMB, which is calculated by target sequencing panel.

Since target sequencing test is a laboratory developed test (LDT) and used in clinical practice, it is necessary to validate its results with gold standard methods in advance. Based on the results, scientists can decide its clinical implementation. CancerSCAN $^{\mathbb{R}}$ (Twist Biosciences, CA, USA), which is a next generation cancer gene panel, is considered as a pipeline that targets cancer related genes; its clinical efficacy is found to be high in target therapy, which is based on genomic alteration and is used to treat many types of cancer (22). More than 15,000 patients have undergone genome profiling through CancerSCAN $^{\circledR}$ panel till date, and it has been used for analyzing various clinical specimens (23-25). Recently, we have developed TMB calculation algorithms by using CancerSCAN ${ }^{\circledR}$. In this study, we analyzed the efficacy with which CancerSCAN (pTMB) could detect TMB in NSCLC samples, and we compared it with TMB calculated by WES method (wTMB).

\section{RESULTS}

\section{Exploratory analysis using reference materials}

As an initial step, we compared the outcomes of pTMB and wTMB by using reference samples, whose TMB value $(n=3)$ was already known. The average sequencing depth was above $750 \times$, and the duplication rate was also stable at an average value of $16.3 \%$ (Supplementary Table 1). When the first set was sequenced and analyzed in CancerSCAN, the absolute number of variants identified from each sample were as follows: 8, 18, and 23. When this set was divided by the total target coding region 1.1 Mb, the TMB scores were found to be $6.9,15.5$, and 19.8 (Supplementary Table 2). Using three identical samples, WES method identified the absolute number of variants as 280,560 , and 721 , respectively in the three samples, and it processed wTMB as $8.43,16.86$, and 21.7 , respectively, after being divided by the total target region of $33 \mathrm{Mb}$. The processed pTMB from the duplicated second set was 8.6, 19.8, and 22.4. Similarly, the processed WTMB from the duplicated second set was 8.1, 16.0, and 21.7, respectively. By comparing the data from both the initial and the duplicated dataset, we found that the concordance rate between $\mathrm{pTMB}$ and wTMB was $R^{2}=0.887$ (Fig. 1).

\section{The concordance of TMB in clinical samples}

The clinical samples were obtained by surgically resecting NSCLC $(n=100)$ tissues; these samples were sequenced with CancerSCAN at a mean depth of $1228.6 \times$ (Supplementary Table 3 ). In all the specimens, the tumor purity value was found to be more than $30 \%$ in pathological laboratory. Moreover, when the tumor purity value was calculated using the actually produced sequencing data, it was found to be very high at an average value of more than $80 \%$. On an average, the on-target sequencing coverage was found to be $68.9 \%$. The WES method was conducted on the same specimens and matched with normal samples; the average coverage was $209.4 \times$ from tumor sample and $68.0 \times$ from normal sample. The pre-defined cutoff value for high TMB were as follows: $10 \mathrm{mut} / \mathrm{Mb}$ for wTMB and $16 \mathrm{mut} / \mathrm{Mb}$ for pTMB. Based on these values, clinical samples were categorized as either high TMB (TMB-h) or low TMB (TMB-I). The raw data was presented on the scatter plot, and it

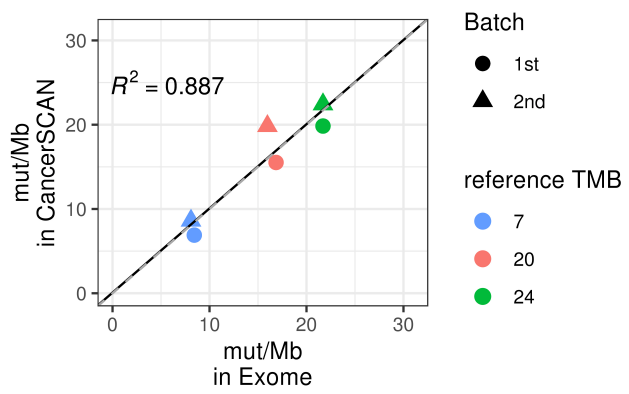

Fig. 1. A comparison of the tumor mutation burden (TMB), which was calculated by CancerSCAN and whole exome sequencing by using reference sample known to harbor TMB of 7, 20, 24 per megabase in number. The $X$-axis indicates the number of TMB determined by exome (mut/Mb). The $\mathrm{Y}$-axis indicates the number of TMB determined by CancerSCAN (mut/Mb). A high correlation was found between replicates produced in the two batches $\left(R^{2}=\right.$ 0.887). Blue color indicates a sample with $T M B$ of $7 \mathrm{mut} / \mathrm{Mb}$, red color indicates a sample with $\mathrm{TMB}$ of $20 \mathrm{mut} / \mathrm{Mb}$, and green color indicates a sample with TMB of 24 mut/Mb. Circles and triangles represent each replicate. 
showed a positive correlation between samples $\left(R^{2}=0.71\right.$, Fig. 2). In terms of categorization, most of the samples $(92.0 \%)$ showed no discrepancy between PTMB and WTMB. However, 8 samples $(8.0 \%)$ were found to be as TMB-h by wTMB method and as TMB-I by pTMB method. Among the concordant samples $(\mathrm{n}=92)$, TMB-h ratio was found to be $8.7 \%(8$ out of 92 samples). We reviewed the 8 cases, which were underestimated by $\mathrm{pTMB}$. In most cases, a relatively higher number of mutations were detected in the genes that were not included in CancerSCAN method (pTMB). As shown in Fig. $3 \mathrm{~B}$, representative genes were the ones marked in orange (described in detail later).

An additional analysis was conducted to determine the tumor purity pathologically, the histology subtypes, and the differentiation of tumor tissues. The concordance between PTMB and $\mathrm{WTMB}$ was accurately found to be more than $95.0 \%$. The high concordance was observed regardless of the surgical stage (Supplementary Table 4).

\section{A comparison of common target regions covered by both WES and CancerSCAN}

By directly comparing the values of pTMB and $W T M B$, we found that the number of WTMB were slightly lower than that of pTMB (Fig. 2, $R^{2}=0.71$ ). To evaluate the difference between pTMB and $W T M B$, we compared the ratio of the coding region by panel sequencing and whole exome sequencing (3.5\%). In addition, we also calculated the ratio of variants identified by target sequencing and WES in the entire population (4.8\%) (Fig. 3A). Based on this result, we inferred that the mutation detected with target sequencing was found to be relatively more than that detected with WES.

As a further step, we compared the number of mutations of each gene by using WES and target sequencing panel (Fig. 3B).
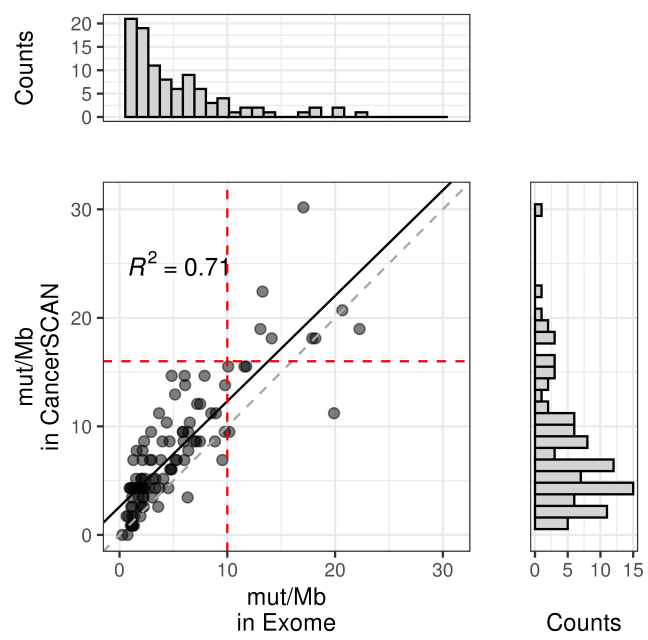

Fig. 2. Scatter and distribution plot of processed $T M B$ (mutation per Mega), which were calculated from CancerSCAN and WES.
We ranked the top 50 genes based on the number of mutations identified by WES. Although most of the genes included here were not related to cancer, the list included five genes that were also identified by target sequencing panel. Last but not the least, we determined the variant allele frequency (VAF) and the number of mutations found by both target sequencing panel and WES, which was based on the variant type (Fig. 3C). The patterns of mutation were generally found to be similar in both the platforms, which showed the highest frequency of missense mutation.

Finally, we investigated the expected CancerSCAN TMB value, which was determined by comparing WES results with CancerSCAN panel content (Supplementary Table 5). The tumor samples sequenced by WES were classified as TMB-high or TMB-low, and $10 \mathrm{mut} / \mathrm{Mb}$ was used as the cutoff value. By using the same method for comparing WES results with that of CancerSCAN panel content, the expected values of TMB-high and TMB-low classifications were found to be similar to the experimentally determined values.

\section{DISCUSSION}

Several evidences indicate that TMB is a predictive biomarker related to $\mathrm{ICl}$ in several types of cancer (3). Moreover, TMB is now considered to be a component of the treatment guidelines related to $\mathrm{ICl}$ in some types of solid cancer. However, there is no consensus on how to measure TMB.

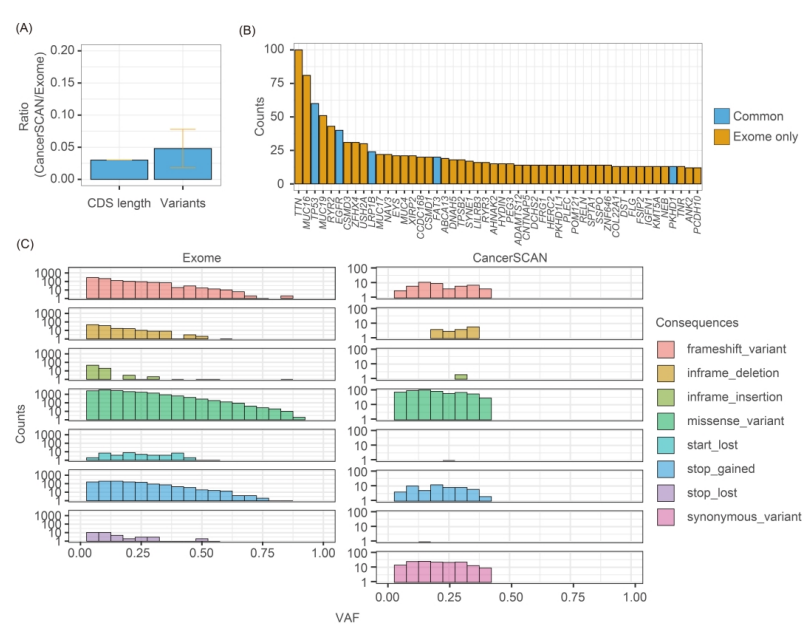

Fig. 3. (A) The ratio of coding region (CDS length), which were analyzed by CancerSCAN and compared with that analyzed by whole exome sequencing (WES), and the ratio of detected variants. When the ratio of variants was higher than the ratio of CDS length, it indicated a relatively higher detection rates of CancerSCAN. (B) The top ranked 50 genes were identified by WES. Orange color indicated a gene that was identified by both CancerSCAN and WES. (C) A comparison of the number of mutations corresponding to the interval of each variant allele frequency (VAF), which was determined by WES and CancerSCAN. 
Compared to WES, which is considered as the gold standard method for TMB calculation, target sequencing offers more benefits in terms of shorter turnaround time and cost-effectiveness. However, there are various technical differences among the two target sequencing platforms, including the number of genes, the coverage of sequence, the variant calling algorithms, etc; therefore, it is difficult to compare the efficacy of the two methods. In particular, each component directly impacts the number of variants identified from the sample, which consequently causes differences in the TMB values of the two methods. Last but not the least, different algorithms may be used to filter out the irrelevant variants associated with TMB calculation; these algorithms differ according to the characteristics of each target sequencing panel. Therefore, an extensive validation of LDT target sequencing panel must be conducted with the standard method. Hence, WES should be performed before clinically selecting patients, who are most likely to benefit from ICl.

In this study, we evaluated the efficacy of LDT target sequencing panel and CancerSCAN by detecting a sample with high mutation burden and comparing it with the WES results of surgically resected NSCLC $(n=100)$ samples. The CancerSCAN technique was applied at a clinical level to determine the genomic alterations of target therapy (26-33). In addition, CancerSCAN results showed that a definite number of mutations could be identified and processed as TMB for references. The CancerSCAN's panel target was $1.1 \mathrm{Mb}$, and it identified the variant according to an annotation database, which presented ethnicity specific mutation without matching normal samples. Since this panel was initially designed for cancer patients, most of the genes included in the panel were cancer-related genes, such as the oncogene and tumor suppressor genes. In addition, the panel was also developed to identify the variants with low VAF. This implies that more mutation, which was missed with a coverage of $100 \times$ to $200 \times$, was captured in this experiment. All these factors are considered as key elements, which consequently caused a discrepancy between PTMB and wTMB values, thereby compelling us to make a direct comparison with the standard method.

In this study, we observed a high correlation between pTMB and $w T M B$ values $\left(R^{2}=0.71\right)$. For the high TMB values detected by panel sequencing and WES, the concordance was found to be $93.0 \%$ for the samples. For the analysis, we made several stepwise approaches and the assumption was made in advance. Firstly, several exploratory analyses were made to evaluate the potential bias. We tested the technical issue by using known TMB values of reference samples in advance. Secondly, we used higher cut-off values for the pTMB. The various cut-off values were proposed from a number of studies, which tested different cancer types with different methods (Supplementary Table 6) $(15,20,34,35)$. These findings indicate that it is challenging to define a standardized cut-off value for high TMB because of the unique components among variable individual panels. The same issue was faced during the fine-tuning of CancerSCAN, that is, the TMB based on HLA was adjusted after considering the characteristics of the patient (20). Hence, we set a relatively high cutoff value of 16 or more mutations per megabase, for the identification of TMB-h patients. Lastly, the variant filtering process was performed in seven steps, which were used for the calculation of TMB.

This study has some limitations. Our samples were acquired from surgically resected tissues, whose clinical response data related to $\mathrm{ICl}$ was limited. In addition, the samples used for this study were pre-selected as histologic, and their tumor purity was pathologically found to be more than $30 \%$. Therefore, interpretation was difficult in samples of low purity. Although we considered factors that affected TMB calculation, such as sample type, cancer type, and sequencing technique, our study established that TMB of well curated LDT was based on pre-defined criteria, which can be used as an alternative to WES method.

\section{MATERIALS AND METHODS}

\section{Specimen}

As an exploratory approach, three types of TMB reference materials were provided by SeraCare (https://www.seracare.com/): one TMB-low sample, expected to have $7 \mathrm{mut} / \mathrm{Mb}$, and two TMB-high sample, expected to have 20 and $26 \mathrm{mut} / \mathrm{Mb}$, respectively. Theses reference samples were designed using human lung cancer cell line with minimum tumor requirement of at least $30 \%$. Reference samples were processed as formalin-fixed paraffin embedded (FFPE) sample sectioned at a 10 um thickness. DNA were extracted from FFPE according to the manufacturer's protocol and duplicated to evaluate the reproducibility.

As a validation process using clinical samples, the fresh samples obtained from NSCLC $(n=100)$ were used. Theses specimens were acquired from surgically resected fresh tissue which were deidentified and stored in the tissue-bank. The surgical stage of samples based on AJCC $8^{\text {th }}$ TNM stage were IVA $(n=1)$, IIIB $(n=10)$, IIIA $(n=23)$, IIB $(n=29)$, IIA $(n=8)$, IB $(n=24), I A(n=5)$. The samples are comprised of both squamous cell carcinoma $(n=30)$ and adenocarcinoma $(n=70)$.

\section{Library preparation}

For the TMB calculation, the laboratory development test (LDT) target sequencing panel, CancerSCAN ${ }^{\circledR}$ v3.0 (K-Master panel), and whole exome sequencing were conducted using same sample. The CancerSCAN ${ }^{\mathbb{R}}$ v3.0 is a hybrid capture panel (a customed panel of Twist Biosciences, Twist Biosciences, CA, USA) which targets an average of 800 sequencing coverage around $1.73 \mathrm{Mbp}$ (22). CancerSCAN ${ }^{\mathbb{R}}$ v3.0 is designed to 407 exomes of genes as well as 3 introns of genes for fusion hotspot and incorporate about 4,000 additional single-nucleotide polymorphism (SNPs) almost evenly located in chromosomes for copy number variation (CNV) purity correction and particular regions for microsatellite instability (MSI) detection. The 
Table 1. Variant filtering steps for the TMB calculation using CancerSCAN v3.0

\begin{tabular}{cll}
\hline Steps & \multicolumn{1}{c}{ Category } & \multicolumn{1}{c}{ Filter out criteria } \\
\hline 1 & Consequence of variants & Non-coding region with splice site \\
2 & Chromosomal location & Mitochondrial DNA \\
3 & Variants allele frequency (VAF) & LowVAF $<0.05$ or HighVAF $>0.4$ \\
4 & Supporting reads & Reads $\leq 4$ \\
5 & Clinical significance & Benign \\
7 & Minor allele frequency & gnomAD $\geq 0.0001$ or \\
& Strand bias between forward and reverse reads & P value $\geq 0.05$ by Fisher's exact test
\end{tabular}

total area targeted by the panel is $1.73 \mathrm{Mbp}$, but only $1.1 \mathrm{Mbp}$, the coding region, was used for the TMB calculation. Whole exome captures were prepared using the Twist human core exome kits (Twist Biosciences). Sequencing was performed by Illumina NextSeq550 (Illumina Inc., CA, USA) after library preparation (Supplementary Table 7).

\section{Alignment and variant calling \\ Sequence reads were mapped to the human genome (hg19) using the Burrows-Wheeler Aligner (26). Duplicate read removal was conducted using Picard tools (https://broadinstitute.github. io/picard/) and SAMtools (27) and the local alignment was optimized by The Genome Analysis Toolkit (28). For the de- tection of single nucleotide variants of CancerSCAN ${ }^{\mathbb{R}}$, results of two types of variant callers (MuTect (29), and LoFreq (30)) were used and integrated to increase sensitivity, particularly for the low VAF variants. Pindel (31) was used to detect indels. For the detection WES, MuTect and Pindel were used.}

\section{Calculation of tumor mutation burden}

We used pre-defined step wise criteria to evaluate the TMB from CancerSCAN ${ }^{\mathbb{R}}$ (Table 1). All variants including both synonymous and nonsynonymous variants in the coding region, exonic region in other word, were included. This strategy enables to overcome the limitation coming from the small number of panels. In addition, due to the reason that CancerSCAN ${ }^{\circledR}$ targets only tumor tissue without matched normal sample using a deep sequencing method targeting up to $800 \times$, it was inevitable that low variant allele frequency (VAF) variants or germline variants could be included to the result. To filter out the germline variant, we used public and in-house database (22). Based on the previous reports (19), TMB is typically defined as the number of coding mutations including base substitution, and insertion and deletion (indel) per Mega-base (Mb) of the genome examined. The number of genomic alterations after the filtering process were divided by the $33 \mathrm{Mb}$ for WES derived results and $1.1 \mathrm{Mb}$ for CancerSCAN ${ }^{\mathbb{R}}$ derived results.

For the determination of TMB high and low for the WES, we adopted cutoff value of TMB-high, 10 mutations per Mbps, used for the the US Food and Drug Administration approval of pembrolizumab for solid cancer. For the cut-off value of TMB high and low for the CancerSCAN, we set a higher cutoff $(\geq$ $16 \mathrm{mut} / \mathrm{Mbp}$ ) based on the observation that described that the misclassification rate of TMB-High increased with decreasing coding sequence region and increased sequencing depth in panel sequencing $(17,19)$.

\section{ACKNOWLEDGEMENTS}

This study was supported by Bristol Myers Squibb. The biospecimens of this study were provided by Samsung Medical Center BioBank (2019-0029).

\section{FUNDING}

This work was supported Bristol Myers Squibb.

\section{CONFLICTS OF INTEREST}

C. Lee is a senior researcher at Geninus. NKD. Kim is a director at Geninus. WY. Park is a chief executive officer at Geninus Inc. No potential conflicts of interest were disclosed by the other authors.

\section{REFERENCES}

1. Shen $\mathrm{T}$, Pajaro-Van de Stadt $\mathrm{SH}$, Yeat $\mathrm{NC}$ and Lin JC (2015) Clinical applications of next generation sequencing in cancer: from panels, to exomes, to genomes. Front Genet 6,215

2. Luthra R, Chen H, Roy-Chowdhuri S and Singh RR (2015) Next-generation sequencing in clinical molecular diagnostics of cancer: advantages and challenges. Cancers (Basel) 7, 2023-2036

3. Ettinger DS, Wood DE, Aisner DL et al (2021) NCCN Guidelines insights: non-small cell lung cancer, version 2.2021. J Natl Compr Canc Netw 19, 254-266

4. Drilon A, Wang L, Arcila ME et al (2015) Broad, hybrid capture-based next-generation sequencing identifies actionable genomic alterations in lung adenocarcinomas otherwise negative for such alterations by other genomic testing approaches. Clin Cancer Res 21, 3631-3639 
5. Surrey LF, MacFarland SP, Chang F et al (2019) Clinical utility of custom-designed NGS panel testing in pediatric tumors. Genome Med 11, 32

6. Reck M, Rodriguez-Abreu D, Robinson AG et al (2016) Pembrolizumab versus chemotherapy for PD-L1-positive non-small-cell lung cancer. N Engl J Med 375, 1823-1833

7. Larkin J, Chiarion-Sileni V, Gonzalez R et al (2019) Fiveyear survival with combined nivolumab and ipilimumab in advanced melanoma. N Engl J Med 381, 1535-1546

8. Alexandrov LB, Nik-Zainal S, Wedge DC et al (2013) Signatures of mutational processes in human cancer. Nature 500, 415-421

9. Yarchoan M, Hopkins A and Jaffee EM (2017) Tumor mutational burden and response rate to PD-1 inhibition. N Engl J Med 377, 2500-2501

10. Snyder A, Makarov V, Merghoub T et al (2014) Genetic basis for clinical response to CTLA-4 blockade in melanoma. N Engl J Med 371, 2189-2199

11. Rizvi NA, Hellmann MD, Snyder A et al (2015) Cancer immunology. Mutational landscape determines sensitivity to PD-1 blockade in non-small cell lung cancer. Science 348, 124-128

12. Rizvi H, Sanchez-Vega F, La K et al (2018) Molecular determinants of response to anti-programmed cell death (PD)-1 and anti-programmed death-ligand 1 (PD-L1) blockade in patients with non-small-cell lung cancer profiled with targeted next-generation sequencing. J Clin Oncol $36,633-641$

13. Andre T, Shiu KK, Kim TW et al (2020) Pembrolizumab in microsatellite-instability-high advanced colorectal cancer. N Engl J Med 383, 2207-2218

14. Marabelle A, Fakih M, Lopez J et al (2020) Association of tumour mutational burden with outcomes in patients with advanced solid tumours treated with pembrolizumab: prospective biomarker analysis of the multicohort, open-label, phase 2 KEYNOTE-158 study. Lancet Oncol 21, 1353-1365

15. Chalmers ZR, Connelly CF, Fabrizio D et al (2017) Analysis of 100,000 human cancer genomes reveals the landscape of tumor mutational burden. Genome Med 9, 34

16. Le DT, Uram JN, Wang H et al (2015) PD-1 Blockade in tumors with mismatch-repair deficiency. $\mathrm{N}$ Engl J Med $372,2509-2520$

17. Budczies J, Allgauer M, Litchfield K et al (2019) Optimizing panel-based tumor mutational burden (TMB) measurement. Ann Oncol 30, 1496-1506

18. Golkaram M, Zhao C, Kruglyak K, Zhang S and Bilke S (2020) The interplay between cancer type, panel size and tumor mutational burden threshold in patient selection for cancer immunotherapy. PLoS Comput Biol 16, e1008332

19. Hong $\mathrm{TH}$, Cha $\mathrm{H}$, Shim JH et al (2020) Clinical advantage of targeted sequencing for unbiased tumor mutational burden estimation in samples with low tumor purity. J Immunother Cancer 8, e001199

20. Shim JH, Kim HS, Cha $\mathrm{H}$ et al (2020) HLA-corrected tumor mutation burden and homologous recombination deficiency for the prediction of response to PD-(L)1 blockade in advanced non-small-cell lung cancer patients. Ann Oncol 31, 902-911

21. Heydt C, Rehker J, Pappesch R et al (2020) Analysis of tumor mutational burden: correlation of five large gene panels with whole exome sequencing. Sci Rep 10, 11387

22. Shin HT, Choi YL, Yun JW et al (2017) Prevalence and detection of low-allele-fraction variants in clinical cancer samples. Nat Commun 8, 1377

23. Kim ST, Kim KM, Kim NKD et al (2017) Clinical application of targeted deep sequencing in solid-cancer patients and utility for biomarker-selected clinical trials. Oncologist 22, 1169-1177

24. Lee J, Kim ST, Kim K et al (2019) Tumor genomic profiling guides patients with metastatic gastric cancer to targeted treatment: the VIKTORY umbrella trial. Cancer Discov 9, 1388-1405

25. Kim Y, Lee B, Shim JH et al (2019) Concurrent genetic alterations predict the progression to target therapy in EGFR-mutated advanced NSCLC. J Thorac Oncol 14, 193202

26. Lee J, Shim JH, Park WY et al (2019) Rare mechanism of acquired resistance to osimertinib in Korean patients with EGFR-mutated non-small cell lung cancer. Cancer Res Treat 51, 408-412

27. Yun JW, Bae YK, Cho SY et al (2019) Elucidation of novel therapeutic targets for acute myeloid leukemias with RUNX1-RUNX1T1 fusion. Int J Mol Sci 20, 1717

28. Park YH, Shin HT, Jung HH et al (2015) Role of HER2 mutations in refractory metastatic breast cancers: targeted sequencing results in patients with refractory breast cancer. Oncotarget 6, 32027-32038

29. Kim KH, Kim J, Park H et al (2020) Parallel comparison and combining effect of radiomic and emerging genomic data for prognostic stratification of non-small cell lung carcinoma patients. Thorac Cancer 11, 2542-2551

30. Ku BM, Bae YH, Lee KY et al (2020) Entrectinib resistance mechanisms in ROS1-rearranged non-small cell lung cancer. Invest New Drugs 38, 360-368

31. Park S, Ku BM, Jung HA et al (2020) EGFR C797S as a resistance mechanism of lazertinib in non-small cell lung cancer with EGFR T790M mutation. Cancer Res Treat 52, 1288-1290

32. Lee HS, Kim E, Lee J et al (2021) Profiling of conditionally reprogrammed cell lines for in vitro chemotherapy response prediction of pancreatic cancer. EBioMedicine 65, 103218

33. Lee Y, Lee S, Sung JS et al (2021) Clinical application of targeted deep sequencing in metastatic colorectal cancer patients: actionable genomic alteration in K-MASTER project. Cancer Res Treat 53, 123-130

34. Wu HX, Wang ZX, Zhao Q et al (2019) Tumor mutational and indel burden: a systematic pan-cancer evaluation as prognostic biomarkers. Ann Transl Med 7, 640

35. Fancello L, Gandini S, Pelicci PG and Mazzarella L (2019) Tumor mutational burden quantification from targeted gene panels: major advancements and challenges. J Immunother Cancer 7, 183 